\title{
Tendência do número de casos da tuberculose no estado de Minas Gerais
}

\section{Trend in the number of cases of tuberculosis in the state of Minas Gerais}

\author{
Felipe Augusto Fernandes ${ }^{1}\left[\right.$, Isolina Aparecida Vilas Bôas ${ }^{1}$ (D) , Tales Jesus Fernandes ${ }^{2}$ (1)
}

1. Doutorando(a) do Programa de pós-graduação em Estatística e Experimentação Agropecuária do Departamento de Estatística da Universidade Federal de Lavras (UFLA), Minas Gerais, Brasil. 2. Docente do Programa de pós-graduação em Estatística e Experimentação Agropecuária do Departamento de Estatística da Universidade Federal de Lavras (UFLA), Minas Gerais, Brasil.

\section{Resumo}

Objetivo: mostrar uma análise do comportamento e da previsão da série referente aos casos confirmados de tuberculose (TB), notificados no sistema de informação de agravos de notificação no estado de Minas Gerais (MG). Métodos: análise de séries temporais e estudo do efeito da sazonalidade, tendência e intervenção. Os dados são mensais relativos ao número de casos confirmados de tuberculose, concernente aos anos de 2001 a 2019 , que foram retirados do site DATASUS. Resultados: ajustou-se um modelo SARIMA $(1,1,0)(1,0,0)_{12}$, o qual o mais adequado foi o que considerou as possíveis intervenções na série. Conclusão: não deve haver um aumento nem queda brusca no número de casos de TB em MG no ano de 2020.

Palavras-chave: Análise de intervenção; Modelo SARIMA; Tuberculose.

\begin{abstract}
Objective: to show an analysis of the behavior and forecast of the series referring to confirmed cases of tuberculosis (TB), notified in the information system for notification of diseases in the state of Minas Gerais (MG). Methods: analysis of time series and study of the effect of seasonality, trend and intervention. The data are monthly regarding the number of confirmed cases of tuberculosis, concerning the years 2001 to 2019, which were removed from the DATASUS website. Results: a model SARIMA(1,1,0)(1,0,0) ${ }_{12}$ was adjusted, the most appropriate being the one that considered the possible interventions in the series. Conclusion: there should not be an increase in the sharp drop in the number of TB cases in MG in 2020.
\end{abstract}

Keywords: Intervention Analysis; SARIMA Model; Tuberculosis.

\section{INTRODUÇÃO}

A tuberculose (TB) é uma doença infecciosa, ou seja, transmitida por um microrganismo. No corpo humano, o órgão mais afetado geralmente são os pulmões, embora possa acometer outros órgãos e/ou sistemas. A doença é causada pelo Mycobacterium tuberculosis ou bacilo de Koch. Essa doença ainda figura como um sério e desafiador problema de saúde pública global. Só no ano de 2018, cerca de dez milhões de pessoas adoeceram por tuberculose e 1,5 milhão de pessoas, no mundo, morreram em decorrência dela, sendo a TB a principal causa de morte por um único agente infeccioso, ficando na frente do HIV/AIDS ${ }^{1,2}$.

O que se tem visto, em geral, é que o número estimado de casos de TB tem aumentado, e, na tentativa de amenizar o impacto epidemiológico, o governo brasileiro tem aumentado as ações de controle da doença por meio de políticas e programas de saúde. Essas ações de controle da TB dependem de que os gestores do setor de saúde realizem, de maneira eficiente e rotineira, as atividades de identificação precoce dos casos novos, rápida intervenção em grupos de maior risco, adesão adequada ao tratamento e prevenção do óbito, especialmente nas áreas de maior transmissão da doença ${ }^{3,4}$. Ademais, é de grande relevância uma vigilância epidemiológica efetiva para orientar decisões do tratamento, uma vez que essa doença pode apresentar-se de forma grave, levando o paciente acometido a tratamentos de até 21 meses e passando por várias fases terapêuticas e efeitos colaterais, às vezes, graves ${ }^{5}$.

No Brasil, o perfil epidemiológico da TB é dominado por homens na faixa etária de 31 a 40 anos, analfabetos, sem acesso aos serviços básicos de saúde e de baixo poder aquisitivo, visto que tal patologia é fortemente associada a comorbidades como etilismo, tabagismo, HIV/AIDS e diabetes. A situação supracitada se agrava devido à grande expansão territorial e o clima tropical do país, o qual possui características que influenciam diretamente no surgimento de doenças, bem como na sua propagação.

O estado de Minas Gerais (MG) está localizado na Região Sudeste do País e é um dos maiores estados brasileiros; dessa forma, o estado retrata bem essa diversificação brasileira. Igualmente aos demais estados que convivem com doenças negligenciadas, em MG, essas também são, em sua maioria, afetadas em razão 
de determinantes sociais ${ }^{6}$.

De acordo com a Secretaria de Saúde do Estado de Minas Gerais $^{7}$, no cenário nacional, MG se encontra como o quinto estado com o maior número de casos no País, com o total de 4.022 casos de TB (casos novos e retratamentos) em 2017. Dos 853 municípios do estado, 538 tiveram, pelo menos, 1 caso de TB notificado entre os seus residentes, totalizando 3.591 casos novos de TB no estado, em 2017. Observa-se que as unidades regionais de saúde de Belo Horizonte, Juiz de Fora, Montes Claros e Coronel Fabriciano apresentaram maior número de casos novos no referido ano, respondendo por $47,7 \%$ do total do estado.

Apesar de o número de incidência de TB em Minas Gerais se manter estável, o número de mortalidades sofreu um considerável aumento, podendo esse fato estar relacionado por descobrirem a doença somente com o óbito, com a ausência da sua notificação enquanto suspeita de caso, ou ainda, por um tratamento ineficaz da doença ${ }^{8}$.

O estudo da série temporal é aplicado em diversas pesquisas, nas mais variadas áreas do conhecimento, como Saúde, Administração, Economia, Ciências Contábeis, Ciências Biológicas, Ciências Ambientais, Ciências Exatas, entre outras. Com isso, a utilização dessas técnicas pode auxiliar as tomadas de decisões governamentais, uma vez que é possível analisar o efeito, quando houver, da sazonalidade, tendência e intervenção no número de casos de TB em MG. Uma série temporal pode ser ajustada por modelos distintos. No estudo da série de TB, optou-se, inicialmente, pelo Autorregressivo-Integrado-Médias Móveis (ARIMA); no entanto, foi necessária a utilização do modelo ARIMA sazonal multiplicativo (SARIMA), uma vez que os dados possuem característica de autocorrelação sazonal.

Este artigo teve como objetivo analisar o comportamento e a previsão da série referente aos casos confirmados de tuberculose, notificados no sistema de informação de agravos de notificação no estado de Minas Gerais, empregando análise de séries temporais e estudando o efeito da sazonalidade, tendência e intervenção.

\section{MÉTODOS}

Os dados são mensais relativos a casos confirmados de tuberculose, notificados no sistema de informação de agravos de notificação no estado de MG, referentes aos anos de 2001 a 2019 e foram retirados do site DATASUS ${ }^{9}$, do Ministério da Saúde. Foram excluídos os registros encerrados com mudança de diagnóstico, uma vez que os períodos disponíveis correspondem aos anos de notificação dos casos.

Inicialmente, foi efetivada uma análise descritiva dos dados, por meio de um Boxplot, considerando as informações anuais, para verificação da ocorrência do número de casos de TB. Em seguida, foi apresentado o gráfico da série temporal da variável em estudo, para posterior ajuste de modelos que explicam a série e façam boas previsões.

Geralmente, toda série temporal, $Y_{t}$, em que $t=1,2, \ldots, \mathrm{n}$ pode ser apresentada como soma $Y_{t}=T_{t}+S_{t}+a_{t^{\prime}}$ em que a tendência $\left(T_{t}\right)$ é um aumento ou diminuição gradual das observações ao longo de um período; a sazonalidade $\left(S_{t}\right)$ mostra flutuações ocorridas em períodos, podendo ser mensal, trimestral, diária, etc. e a componente aleatória ou erro $\left(a_{t}\right)$ mostra as oscilações aleatórias irregulares. A suposição usual é que $a_{t}$ seja uma série exclusivamente aleatória ou ruído branco independente, com média zero e variância constante ${ }^{10}$.

Como na prática, as séries encontradas apresentam tendência e/ou sazonalidade, Box e Jenkins $(1976)^{11}$ propuseram o modelo ARIMA, o qual é apropriado para descrever séries não estacionárias, ou seja, séries que não possuem média constante no período de análise, nas quais os parâmetros quase sempre são pequenos, o que não é o caso deste estudo.

Em geral, o modelo ARIMA examina a autocorrelação entre os valores da série em instantes sucessivos. Os modelos que contemplam as séries que apresentam autocorrelação sazonal são conhecidos como SARIMA, os quais contêm uma parte não sazonal com parâmetros $(p, d, q)$ e uma sazonal com parâmetros $(P, D, Q) \cdot{ }^{10,12}$ Em que, $p$ e $P$ representam a ordem do $A R(p)$, d e $D$ são o número(s) de diferença da série e $q$ e $Q$ a ordem de médias móveis.

Neste estudo, o modelo proposto para descrever o número de casos notificados no sistema de informação de agravos de notificação no estado de $M G$ foi o $\operatorname{SARIMA}(1,1,0)(1,0,0)_{12}$, o primeiro não considerando os pontos de intervenção e, posteriormente, considerando esses pontos; assim têm-se o Modelo 1 e o Modelo 2, respectivamente. Pode-se escrever um modelo SARIMA $(1,1,0)(1,0,0)_{12}$, da seguinte forma:

$$
\left(1-\varphi_{1} B\right)\left(1-\phi_{1} B^{12}\right)(1-B) Y_{t}=a_{t}
$$

Em que:

$B$ é operador de translação para o passado definido como $B^{\text {n }}$ $\mathrm{Y}_{\mathrm{t}}=\mathrm{Y}_{\mathrm{t}-\mathrm{n}}$;

$\varphi(B)=\left(1-\varphi_{1} B-\cdots-\varphi_{P} B^{p}\right) \rightarrow$ Polinômio autorregressivo;

$\phi\left(B^{s}\right)=\left(1-\phi_{1} B^{1 s}-\cdots-\phi_{p} B^{p s}\right) \rightarrow$ Polinômio autorregressivo sazonal.

Para verificar a tendência da série, foi observado seu correlograma; para a presença de sazonalidade, além da análise gráfica do correlograma, foi feito o teste de Kruskal-Wallis, para verificar se a série possui sazonalidade determinística.

Por meio de um gráfico de amplitude-média, será observado se existe dependência entre a amplitude e a média, e analisado o valor $\mathrm{p}$ do teste de hipóteses for $(<0,05)$, rejeita-se $\mathrm{H}_{0}$, ou seja, existe necessidade de fazer uma transformação nos dados 
para estabilizar a variância, sendo a transformação logarítmica a mais apropriada.

Depois de confirmar a presença da componente tendência por meio dos testes, aplica-se a primeira diferença, procedimento que é indicado quando a série apresenta tendência estocástica. Após estimar ou eliminar as componentes tendência (e sazonalidade, se houver) e verificar a estacionariedade da série, realiza-se o ajuste de alguns modelos.

Morettin e Toloi (2004) entendem intervenção como uma ocorrência de algum tipo de evento em dado instante de tempo $\mathrm{T}$, conhecido a priori. Dessa forma, os pontos significativos como possíveis pontos de intervenção serão considerados em um modelo separado. A análise de resíduos foi feita por meio do teste de Ljung, Box e Pierce, que se rejeita $\mathrm{H}_{0}$ : o resíduo do modelo é ruído branco, caso o valor $p$ do teste de hipóteses seja $(<0,05)$.

A partir disso, compararam-se os modelos indicados por dois métodos de qualidade de ajuste: os critérios de informação, critério de informação de Akaike (AIC) e o critério de HannanQuinn (HQC). Prosseguindo com a análise, retiram-se os seis últimos dados referentes a 2019 para realizar a previsão e comparar com os valores reais, a fim de verificar qual deles apresenta os menores valores do Erro Quadrático Médio de Previsão (EQMP) e Média Percentual Absoluta do Erro (MAPE) e, portanto, definir o mais adequado. Em que:

$$
E Q M P=\frac{1}{n} \sum_{i=1}^{n}\left(y_{i}-\hat{y}_{i}\right)^{2} ; M A P E=\frac{1}{n} \sum_{i=1}^{n}\left|\frac{y_{i}-\hat{y}_{i}}{y_{i}}\right| \times 100 \%
$$

Com o modelo selecionado e ajustado, esse pode ser utilizado para realizar as previsões sobre as observações futuras. Todas as análises realizadas foram utilizando o software Gretl (Gretl, 2019) $)^{13}$ e o software R (R Core Team, 2019) ${ }^{14}$.

\section{RESULTADOS}

Para uma análise descritiva dos dados, ver-se, na figura 1, Boxplot para os dados anuais de casos de casos confirmados de TB em MG.

Na figura 2, observa-se o gráfico da série do número de casos de TB em MG, no período de janeiro de 2001 a dezembro de 2019. Nessa figura, percebe-se que a série não é estacionária, ou seja, não possui média zero nem variância constante.

O gráfico amplitude versus média, apresentado na figura 3, permite observar se há necessidade de transformação. As estatísticas amplitude-média para número de casos de TB em MG, usando 19 subamostras de dimensão 12, geram os seguintes dados: inclinação da amplitude versus média $=0,1307$ e valor $p$ para $H_{0}$ : inclinação $=0$ é 0,105007 . Nesse caso, a hipótese nula não é rejeitada, ou seja, a inclinação não é diferente de zero, isto implica que não há necessidade de transformação nos dados.

Figura 01: Boxplot das observações anuais da série do número de casos de TB em MG.

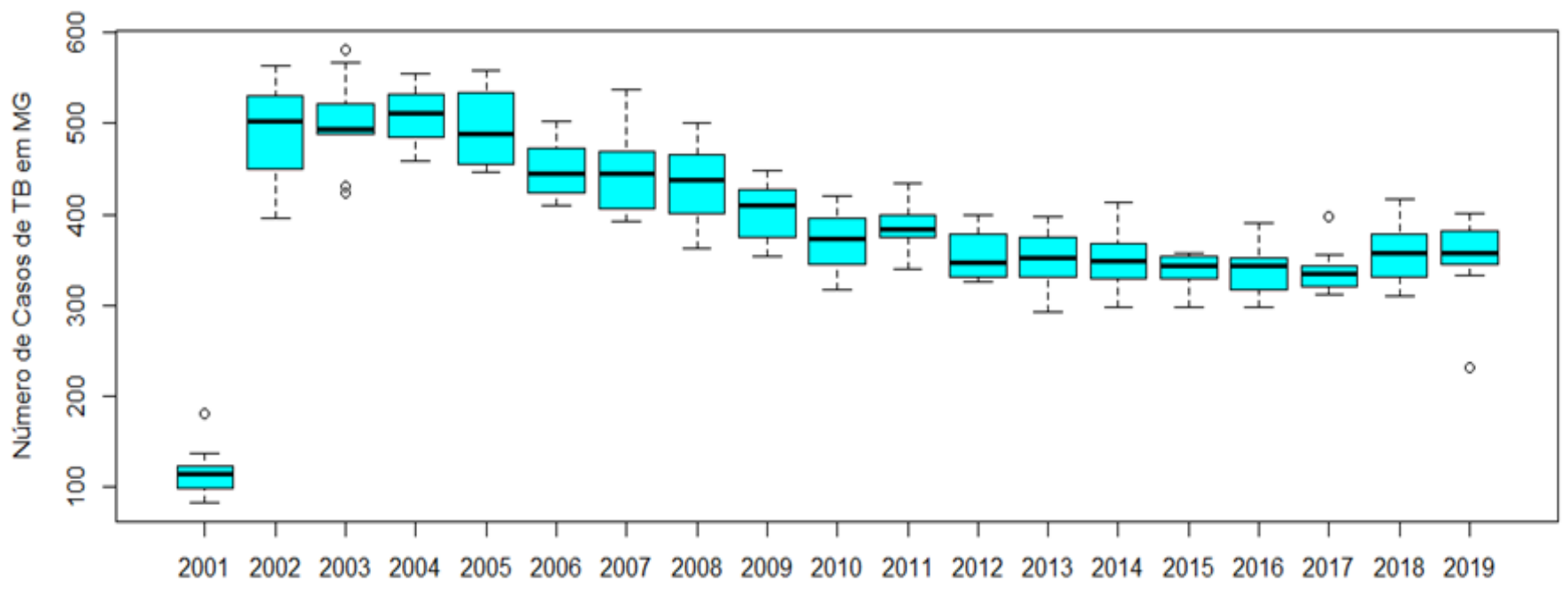


Figura 2. Série do número de casos de TB em MG de janeiro de 2001 a dezembro de 2019.

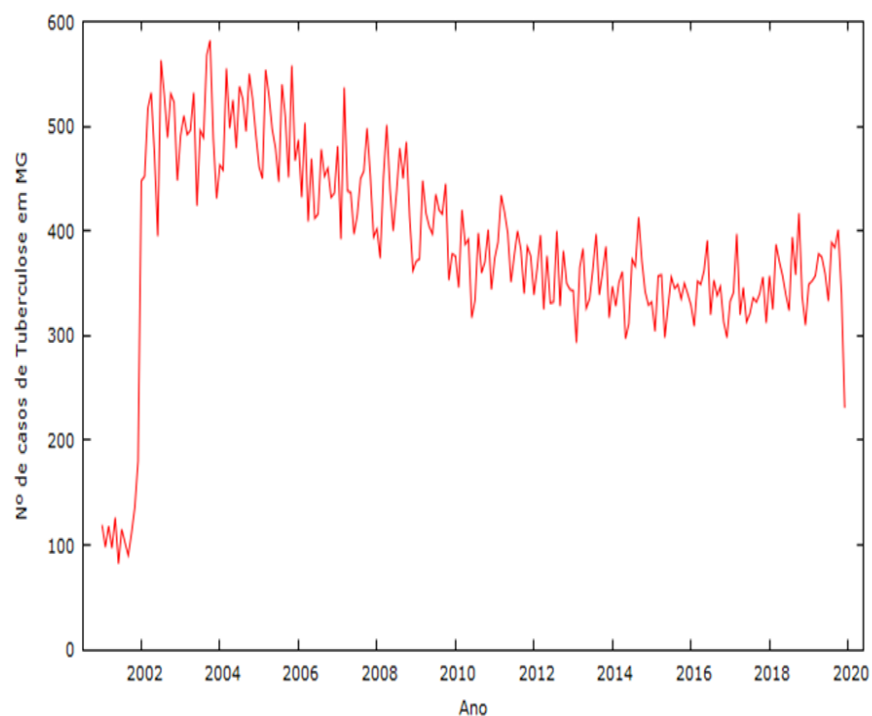

Como a série ainda não era estacionária, foi realizada a primeira diferença, em seguida, feito o teste de Kruskal-Wallis e obteve o valor-p de 0,1122; com isso, conclui-se que a série não possui sazonalidade determinística. Quando a série não apresenta o componente sazonal, mas apresenta a componente tendência, ou esses componentes são homogêneos não estacionários, ela pode ser representada por um modelo ARIMA, ou seja, o ajuste pode ser feito por um modelo sem o componente sazonal ${ }^{15}$.

No entanto, a série apresentou "lags" sazonais, isto é, múltiplos de períodos. Isso significa que há necessidade de se considerar uma sazonalidade estocástica, ou seja, ajustar a série original um modelo ARIMA sazonal $(S A R I M A)^{10}$. Foram ajustados dois modelos, para a série do número de casos de TB em MG, sendo $\operatorname{SARIMA}(1,1,0)(1,0,0)_{12}$ com e sem intervenção.

A seguir é apresentado o ajuste dos dois modelos propostos:

Modelo $1 \operatorname{SARIMA}(1,1,0)(1,0,0)_{12}$, sem intervenção:

$$
(№ T B)=\frac{a_{t}}{(1+0,322582 B)\left(1-0,394085 B^{12}\right)(1-B)}
$$

Modelo $2 \operatorname{SARIMA}(1,1,0)(1,0,0)_{12}$, com intervenção:

$$
\begin{gathered}
\left(N^{\circ} T B\right)=\frac{a_{t}}{(1+0,317289 B)\left(1-0,361258 B^{12}\right)(1-B)}+ \\
\left(112,720 * S^{13}\right)+\left(-109,311 * S^{228}\right)
\end{gathered}
$$

Cabe salientar que, pelo teste de Ljung, Box e Pierce, o resíduo é ruído branco para os dois modelos, com os valores de $p$ de 0,062 e 0,076, para os Modelos 1 e 2, respectivamente. Sendo que o erro padrão dos dois parâmetros de autocorrelação dos modelos 1 e 2 foram inferiores a 0,07, para os pontos de intervenção do Modelo 2, os erros padrões foram de 32,23 e 41,79 para as observações 13 e 228 , respectivamente.
Figura 3. Gráfico da amplitude-média da série do número de casos de TB em MG, de 2001 a dezembro de 2019.

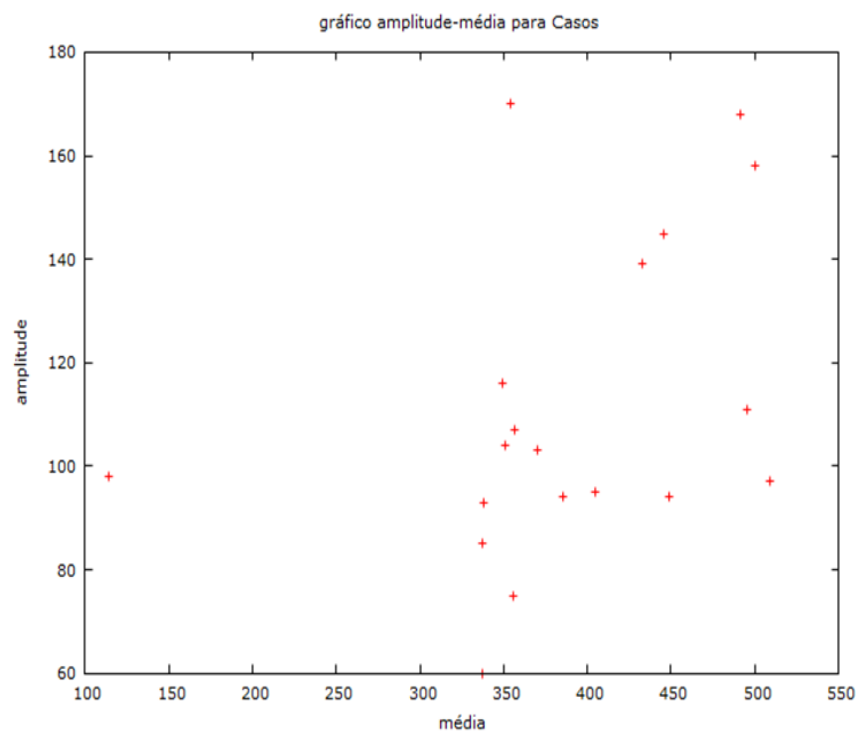

A tabela 1 apresenta os valores dos métodos de qualidade do ajuste para os modelos estudados.

Tabela 1. Modelos ajustados aos dados e seus respectivos valores do Critério de Informação de Akaike (AIC), do Critério Hannan-Quinn (HQ), do Erro Quadrático Médio de Previsão (EQMP) e da Média Percentual Absoluta do Erro (MAPE)

\begin{tabular}{lllll}
\hline Modelo & AIC & HQC & EQMP & MAPE \\
\hline Modelo 1 & 2362,93 & 2367,08 & 2614,47 & $12,26 \%$ \\
Modelo 2 & 2348,57 & 2355,48 & 2651,08 & $12,44 \%$ \\
\hline
\end{tabular}

A figura 4 apresenta as estimativas da série do número de casos de TB em MG com a previsão dada pelo modelo $\operatorname{SARIMA}(1,1,0)$ $(1,0,0)_{12}$ com e sem intervenção.

Figura 4. Série que representa o número de casos de TB em MG, com as estimativas geradas pelos modelos 1 e 2 e as previsões dos dados pelos dois modelos em estudo.

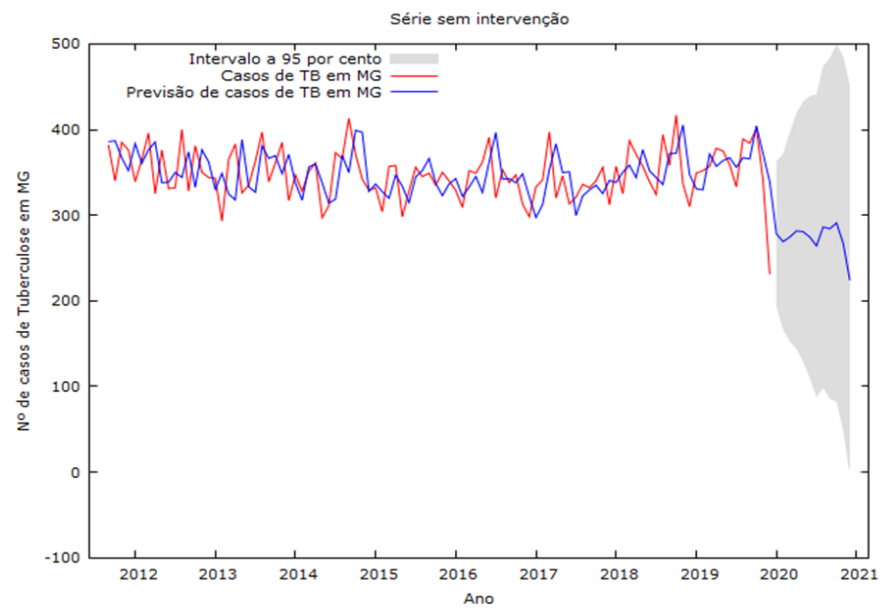




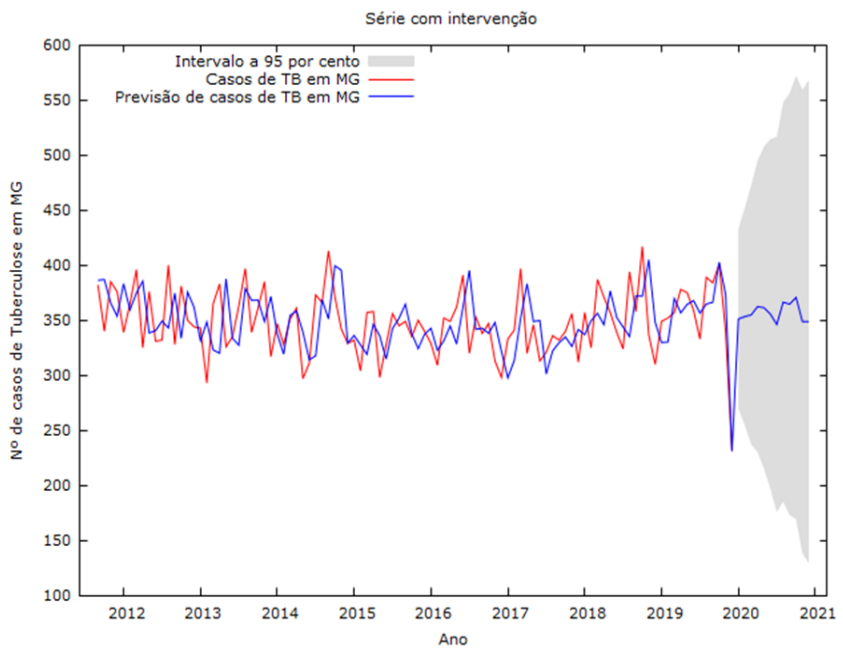

DISCUSSÃO

Em 2001 houve uma subnotificação dos números de casos de TB significativa com relação aos outros anos, mesmo apresentado um mês com outlier bem acima da mediana do ano, o que também foi mostrado em outros trabalhos ${ }^{8,16}$. Uma possível explicação desse baixo número de casos de TB registrados para MG em 2001 é que a taxa de incidência em MG era de 36,6 casos/100 mil habitantes, sendo abaixo da nacional era de 46,5. Neste mesmo período, na Região Oeste de Belo Horizonte, na maior parte dos casos notificados predominou o sexo masculino em uma idade aproximada de 34 anos $^{17}$.

No ano de 2019, o Brasil apresentou 73.864 casos novos de TB, o que correspondeu a um coeficiente de incidência de 35,0 casos/100 mil habitantes. Embora tenha sido observada uma constante tendência de queda entre os anos de 2010 e 2016, o coeficiente de incidência da TB no país aumentou nos anos de 2017 e 2018 em relação ao período anterior ${ }^{1}$. Em MG, percebese esse aumento nos anos de 2018 e 2019, apesar de que, no mês de dezembro de 2019, houve uma queda considerável no número de casos e deve ser analisada com cautela. O relatório da Secretaria de Saúde de Minas Gerais de 2020 deve trazer, de forma clara, o que ocasionou esse fenômeno.

Intervenção é definida como a ocorrência de algum tipo de evento em dado instante de tempo T, conhecido a priori. Tal ocorrência pode manifestar-se por um intervalo de tempo subsequente e que afeta, temporária ou permanentemente, a série em estudo ${ }^{10}$. A inclusão de uma possível intervenção nos dados se deu analisando as figuras 1 e 2, em que se percebe que o ano de 2001 apresenta uma característica diferente na série, e a última observação de 2019 também apresenta uma queda considerável no número de casos.

Os pontos de intervenção significativos do Modelo $2, \mathrm{~S}^{13}$ e $\mathrm{S}^{228}$ representam as datas de janeiro de 2002 (448 casos) e dezembro de 2019 (231 casos), visto que esses pontos supracitados representam os meses que apresentam uma mudança considerável no comportamento da série.
O relatório do sistema nacional de vigilância em saúde, do Ministério da Saúde (2005), que traz a situação de MG, avaliando os anos de 1993 a 2003, não destaca nenhuma justificativa para o baixo número de casos registrados em 2001. O que se pode notar é que a incidência de TB apresentou uma queda a partir do ano de 2000, havendo um aumentou a partir de 2002.

Por meio da tabela 1, observa-se que os modelos apresentaram uma diferença pequena comparando os valores do EQMP e MAPE, sendo que o Modelo 1 apresentou menores valores de EQMP e MAPE, deixando claro que esse modelo possui menor erro considerando as previsões das últimas seis observações em relação aos valores reais.

O Modelo 1 obteve maiores valores do AIC e do HQC, desta maneira, considerando esses critérios de informação supracitados; o Modelo 2 seria o que melhor descreve a série do número de casos de TB em MG.

O paradoxo para a definição do melhor modelo pode ser explicado pelo fato de as intervenções ocorrerem no início e no final da série, e o EQMP e o MAPE considerar apenas as observações finais. Com isso, torna-se interessante a análise dos dois modelos, uma vez que umas das informações mais importantes podem ser extraídas das previsões dos modelos.

Observa-se, na figura 04, que considerar dezembro de 2019 como um ponto de intervenção altera, consideravelmente, a previsão do modelo para o ano de 2020. Visto pela sequência da série, mostra-se razoável para questão de previsão, que a utilização do Modelo 2 seja a mais adequada, uma vez que, a previsão do Modelo 1 mostra uma drástica mudança do comportamento da série sem que haja nenhuma política mais agressiva de combate a TB.

Na epidemiologia, existe a necessidade de predizer o futuro para que haja possíveis intervenções nos processos do presente, dado que isso é mais que mera curiosidade ou interesse mesquinho. Uma vez que, de fato, o assunto envolve vida ou morte, e decisões acertadas impactam na redução da carga de doenças na população ${ }^{18,19}$.

\section{CONSIDERAÇÕES FINAIS}

Os modelos ajustados $\operatorname{SARIMA}(1,1,0)(1,0,0)_{12}$ com e sem intervenção obtiveram resultados próximos em relação aos valores do EQMP e o MAPE, mostrando-se adequados para descrever séries referentes aos casos confirmados de tuberculose, notificados no sistema de informação de agravos de notificação no estado de Minas Gerais (MG).

Observa-se a existência de um componente sazonal de autocorrelação a cada 12 meses em relação ao número de casos de TB em MG, levando ao ajuste de modelo SARIMA.

Entre os modelos ajustados com e sem intervenção, verificou-se 
que o modelo com intervenção fornece informações adicionais, ou seja, no mês de janeiro de 2002, houve um aumento considerável do número de casos de TB em MG e, em dezembro de 2019, houve uma diminuição acentuada da série. E, para fins de previsão, este modelo se mostra mais adequado, já que ressalta que não deve haver um aumento nem queda brusca no número de casos de TB em MG para o ano de 2020.

\section{AGRADECIMENTO}

Ao Conselho Nacional de Desenvolvimento Científico e Tecnológico (CNPq), pela bolsa concedida ao primeiro autor.

\section{REFERÊNCIAS}

1. Ministério da Saúde (BR). Boletim Epidemiológico de Tuberculose 2020. Secretaria de Vigilância em Saúde. Número Especial. Disponível em: http://www. aids.gov.br/pt-br/pub/2020/boletim-epidemiologico-de-turbeculose-2020

2. Silva DR, Mello FCQ, Kritski A, Dalcolmo $M$, Zumla A, Migliori GB. Série tuberculose. J. bras. pneumol. 2018 Mar-Apr; 44(2): 71-72. doi: http://dx.doi. org/10.1590/s1806-37562018000020001

3. Bierrenbach AL, Duarte EC, Gomes ABF, Souza MFM. Tendência da mortalidade por tuberculose no Brasil, 1980 a 2004. Rev. Saúde Pública. 2007 Set; 41(Supl. 1): 15-23. doi: https://doi.org/10.1590/S0034-89102007000800004.

4. Rossetto M, Hesler LZ, Maffacciolli R, Rocha CF, Oliveira DL. Comunicação para promoção da saúde: as campanhas publicitárias sobre tuberculose no Brasil. Rev Enferm UFSM. 2017 Jan-Fev; 7(1): 18-28. doi: https://doi. org/10.5902/2179769222801.

5. Paz M, Argemi X, Schramma F, Mielcarekc M, Kassegne L, Hansmannb $Y$, Fraisse $P$. Tuberculose multirésistante: une prise en charge qui pèse lourd aux hôpitaux universitaires de Strasbourg. Rev Maladies Resp. 2019 Nov; 36(9): 1011-1018. doi: https://doi.org/10.1016/j.rmr.2019.07.006.

6. Carvalho Filho AX, Silva JP. Tuberculose em Minas Gerais e Determinantes Sociais. Atenas Higeia. 2019 Jul-Dez; 1(2): 24-27.

7. Secretaria de Estado de Saúde de Minas Gerais 2019. Plano Estadual pelo fim da Tuberculose como problema de saúde pública em Minas Gerais 2019 2022 [Internet]. Minas Gerais: SUS; 2019 [2019/10/01]. Disponível em: http:// www.saude.mg.gov.br/images/noticias e eventos/000_2019/jane fev mar/ PLANO_ESTADUAL_PELO_FIM_DA_TUBERCULOSE_COMO_PROBLEMA_DE_ SA\%C3\%9ADE_P\%C3\%9ABLICA_EM_MG.pdf.

8. Oliveira A, Rocha BB, Baia LS, Pereira RP, Carvalho DS. Tuberculose: uma abordagem geral dos principais aspectos em Minas Gerais e no Brasil. In: Anais do ISimpósio de Enfermagem da FACIG; 2018 Maio 11-12; Manhuaçu, MG: FACIG; 2018.

9. Ministério da Saúde (BR). DATASUS. Informações de Saúde (TABNET). Disponível em: http://tabnet.datasus.gov.br/cgi/tabcgi.exe?sinannet/cnv/ tubercmg.def

10. Morettin PA, Toloi CMC. Análise de séries temporais. São Paulo: Edgar BlüCher; 2004.

11. Box GP, Jenkins GM. Time series analysis, forecasting and control. San Francisco: Holden-Day; 1976.

12. Espinosa MM, Prado SM, Ghellere M. Uso do modelo SARIMA na previsão do número de focos de calor para os meses de junho a outubro no Estado de Mato Grosso. Ciência e Natura. 2010; 1(2): 07-21. doi: https://doi. org/10.5902/2179460X9482.

13. Gretl, Gnu Regression, Econometrics and Time-series Library [Internet]. 2019 [acesso 2019 Nov 08]. Disponível em: http://gretl.sourceforge.net/.

14. R Core Team. R: a language and environment for statistical computing. Vienna: R Foundation for Statistical Computing, 2016 [Internet]; 2016 [acesso 2019 Nov 8]. Disponível em: https://www.R-project.org..

15. Sáfadi, T. Uso de séries temporais na análise de vazão de água na represa de furnas. Ciênic. agrotec. 2004 Fev; 28(1): 142-148. doi: http://dx.doi. org/10.1590/S1413-70542004000100019.

16. Oliva HNP, Oliveira AG, Godinho ACVCQ, Alves BLR, Ramos MTBP, Galdino VAC, et al. Estudo epidemiológico da tuberculose no estado de Minas Gerais. REAS. 2018; (18): e78. doi: https://doi.org/10.25248/reas.e78.2019.

17. Paixão LMM, Gontijo ED. Perfil de casos de tuberculose notificados e fatores associados ao abandono, Belo Horizonte, MG. Rev. Saúde Pública. 2007 Abr; 41(2): 205-213. doi: https://doi.org/10.1590/S0034-89102007000200006.

18. Antunes JLF, Cardoso MRA. Uso da análise de séries temporais em estudos epidemiológicos. Epidemiol. Serv. Saúde. 2015 Jul-Set; 24(3): 565-576. doi: $10.5123 /$ S1679-49742015000300024.

19. Amaral MTR, Conceição KS, Andrade MG, Padovani CR. Generalized growth curve model for Covid-19 in brazilian states. Rev. Bras. Biom. 2020; 38(3): 125146. doi: $10.28951 /$ rbb.v38i2.481 